\title{
THE X-RAY CORONA AND THE PHOTOSPHERIC MAGNETIC FIELD
}

\author{
A. S. KRIEGER, G. S. VAIANA and L. P. VAN SPEYBROECK \\ American Science and Engineering, Cambridge, Mass., U.S.A.
}

\begin{abstract}
Soft X-ray (3-60 $\AA$ ) photographs of the solar corona have been obtained on four flights of a rocket-borne grazing incidence telescope having a resolution of a few arc seconds. The configuration of the X-ray emitting structures in the corona has been compared to the magnetic field distribution measured by photospheric longitudinal magnetograms. The X-ray structures trace the three dimensional configuration of the magnetic field through the lower corona.

Active regions in the corona take the form of tubular structures connecting regions of opposite magnetic polarity within the same or adjacent chromospheric active regions.

Higher, larger structures link widely separated active regions into complexes of activity covering substantial fractions of the disk. The complexes are separated by areas of low average field in the photosphere. Interconnections across the solar equator appear to originate over areas of preceding polarity.

Enhanced X-ray emission is observed, outside the active belt, over areas of enhanced general magnetic field. Bright point-like X-ray features are observed above bipolar areas in the general coronal field.

Two, probably related, classes of X-ray features are associated with points of high field gradient along the longitudinal magnetic, 'neutral line'. Both in the non-flaring active region and in flares the brightest emission is observed from one or more small line sources (at our present resolution). In flares this can account for a substantial fraction of the total soft X-ray emission of the flare.
\end{abstract}

\section{Introduction}

In order to derive meaningful models of the physical processes taking place in the solar corona, one must know the structure of the corona and the configuration of the magnetic field through the corona. For example, the direction and strength of the magnetic field in the lower corona will determine the type of waves that may propagate there and the mechanism by which they dissipate their energy. Also, the problems of energy storage in non-flaring active regions and energy release in flares require the knowledge of the configuration of the magnetic field of the active region in the lower corona.

Mathematical extension of the photospheric fields into the corona is a difficult exercise. Observationally, all three components of the field must be measured, and theoretically, one must solve for a stable, force-free field configuration. Direct observation of the coronal structures are needed to acquire confidence in the calculations. In visible light, coronal structures can be observed only at the limb, and, with sufficient resolution, only during total solar eclipses, and occasionally, under good seeing conditions, in a few selected lines.

Soft X-rays provide an advantageous waveband for examination of the lower corona. Soft X-rays are thermally emitted by plasmas with temperatures in the range of $10^{6} \mathrm{~K}$ or more, hence they are the characteristic radiation of the coronal plasma. Moreover, there is no photospheric or chromospheric background radiation at these 
wavelengths, therefore, coronal structures can be seen in projection on the solar disk. In addition, X-ray optics with angular resolution approaching that attainable in visible light are now available.

Over the past several years our group has conducted four successful sounding rocket flights of a grazing incidence X-ray telescope with a resolution of a few arc seconds. A full description of the instrumentation has been reported in the literature (Giacconi et al., 1969; Vaiana et al., 1968). We have obtained photographs in the 3 to $60 \AA$ wavelength range of the X-ray emitting structures of the solar corona as projected on the disk. Two of the flights were devoted to the study of solar flares, and the other two were directed primarily toward investigations of the general corona. On all four flights non-flaring active regions were observed.

An examination of an X-ray photograph obtained during our most recent flight together with a map of the photospheric longitudinal magnetic field (Figure 1) shows the highly ordered structures observed in the corona. They are clearly associated with the underlying photospheric fields. It is natural to suppose that the X-ray structures reflect the configuration of the coronal magnetic fields. To make a plausibility argument, one need only say that in order to produce a detectable photographic image with the X-ray telescope currently in use, a coronal region must have a temperature greater than $10^{6} \mathrm{~K}$ and a density greater than a few times $10^{8} \mathrm{~cm}^{-3}$. Thus the highly ordered structures observed in X-rays can be contained by magnetic fields of only a few gauss in strength. Accordingly, we discuss here the relationship between the observed coronal $\mathrm{X}$-ray structures and the underlying photospheric magnetic field in order to understand the configuration of the coronal magnetic field. Other aspects of our X-ray photographs have been discussed elsewhere (Van Speybroeck et al., 1970; Vaiana and Giacconi, 1969).

Although the discussion in this paper is still entirely qualitative the results display the general characteristics of the coronal magnetic field. We have observed X-ray structures associated with a variety of solar phenomena. Active regions appear in the corona to be composed of tubular structures connecting regions of opposite magnetic polarity on the photospheric longitudinal field map. Widely separated active regions are interconnected over significant fractions of the solar disk by X-ray emitting structures. Bright, hot, narrow features have been observed bridging the 'neutral line' of the longitudinal magnetic field in active regions and flares. Relatively diffuse X-ray emitting features overlie areas of enhanced field on the quiescent disk. These features can be identified as the bases of white light coronal structures. Small, bright, X-ray features in the quiescent corona are associated with bipolar areas in the general solar field.

\section{Active Regions}

When active regions are observed in X-rays at the limb of the Sun (Figure 2) they are seen to be complex tubular arches and/or loops of enhanced density and temperature rising to heights of more than $10^{5} \mathrm{~km}$ above the photosphere. Thus, X-ray observations of active regions on the disk are two-dimensional projections of three-dimensional 


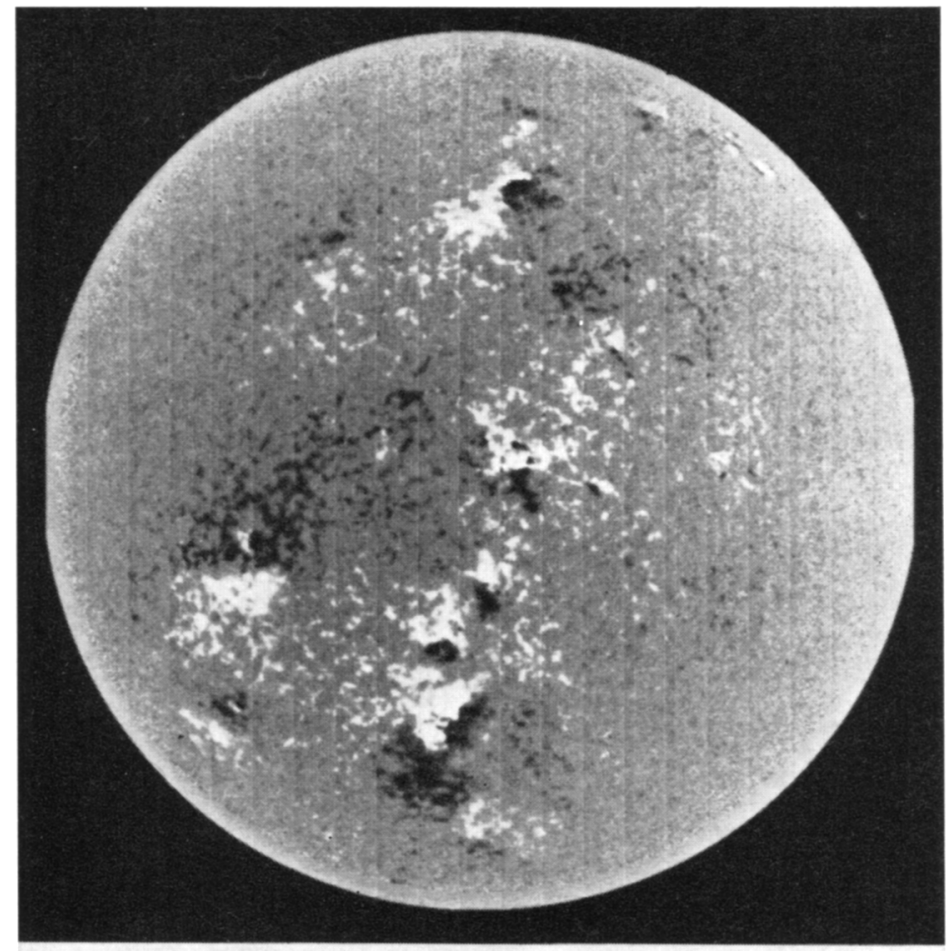

䒿

突응

东

.$\unlhd$

읩

递

ช.

要

氙步

$\times 20$

원

苟要

9

흉

这

证

동

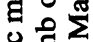

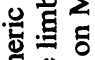

동을 응

表

옴.

\%

है है

즐 융. 을

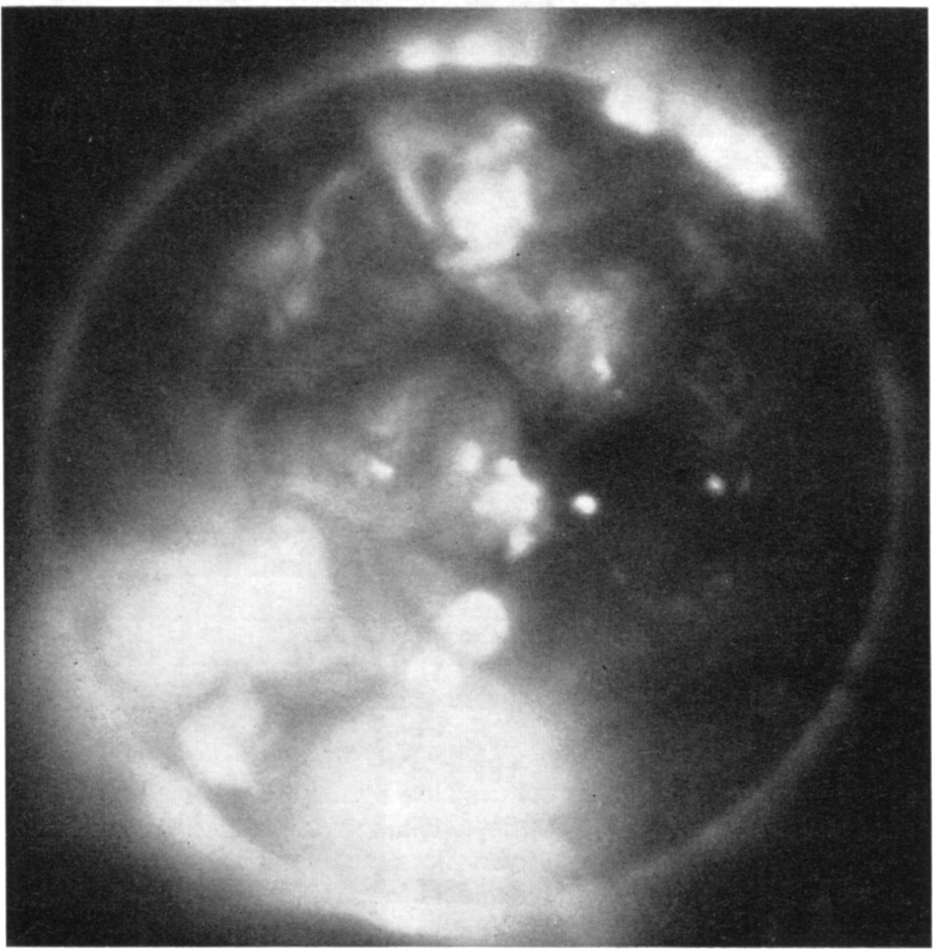

欲焉

峁

흥 염

ఏ

올돈

它

5

政

증

뜐듬

的实

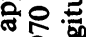

의

ชี

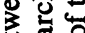

$8{ }^{\circ}$

ธี ฮี ถู

参喜

范

年嵒

$\varangle \&$

- 0 क

我 

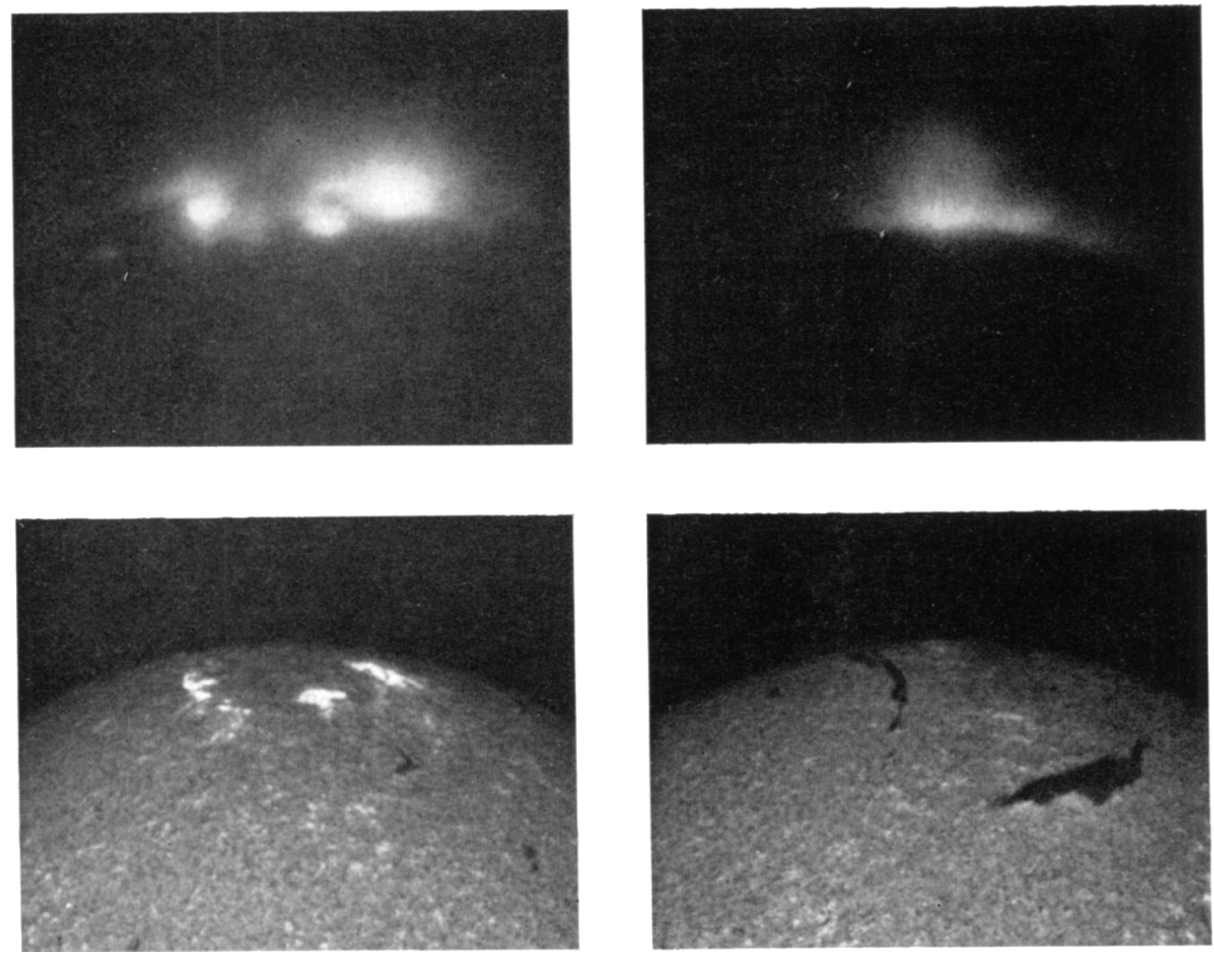

Fig. 2. The appearance of active region structures in X-rays at the limb. Top: X-ray photographs in the 3-17 $\AA$ passband of active region associated coronal features. Left: A group of active regions near the limb. At least three arches connecting different portions of the group can be distinguished. Right: A loop structure associated with an active region very close to the limb. The coronal loop extends to an altitude of at least $150000 \mathrm{~km}$. Bottom: $\mathrm{H} \alpha$ photographs of the corresponding portions of the disk taken two hours before the rocket flight (June 8, 1968) by ESSA Boulder Observatory. The length of the bracket below the photographs is one arc-minute.

stı uctures. The appearance in projection on the disk of the coronal structure of three active regions is shown in Figure 3 together with $\mathrm{H} \alpha$, and $\mathrm{CaK}$ spectroheliograms of the regions, and their longitudinal magnetic field distribution. A close examination of the photographs shows that the structures observed in X-rays interconnect regions of opposite magnetic polarity, as displayed by the longitudinal field map. The interconnections proceed between portions of the same or adjacent active regions. In general, the intensity of the observed X-ray emission is highest near the 'neutral line' of the longitudinal magnetic field.* Often, where a large field gradient is observed, a

* Almost all of our comparisons of the X-ray structures have been made with regard to the longitudinal field maps because of the difficulties involved in obtaining vector magnetic field information. For our purposes here this is satisfactory. However, the reader should remain aware of the ambiguities involved. For example, the location of the longitudinal field 'neutral line' may not be neutral at all, but might possess transverse fields. 

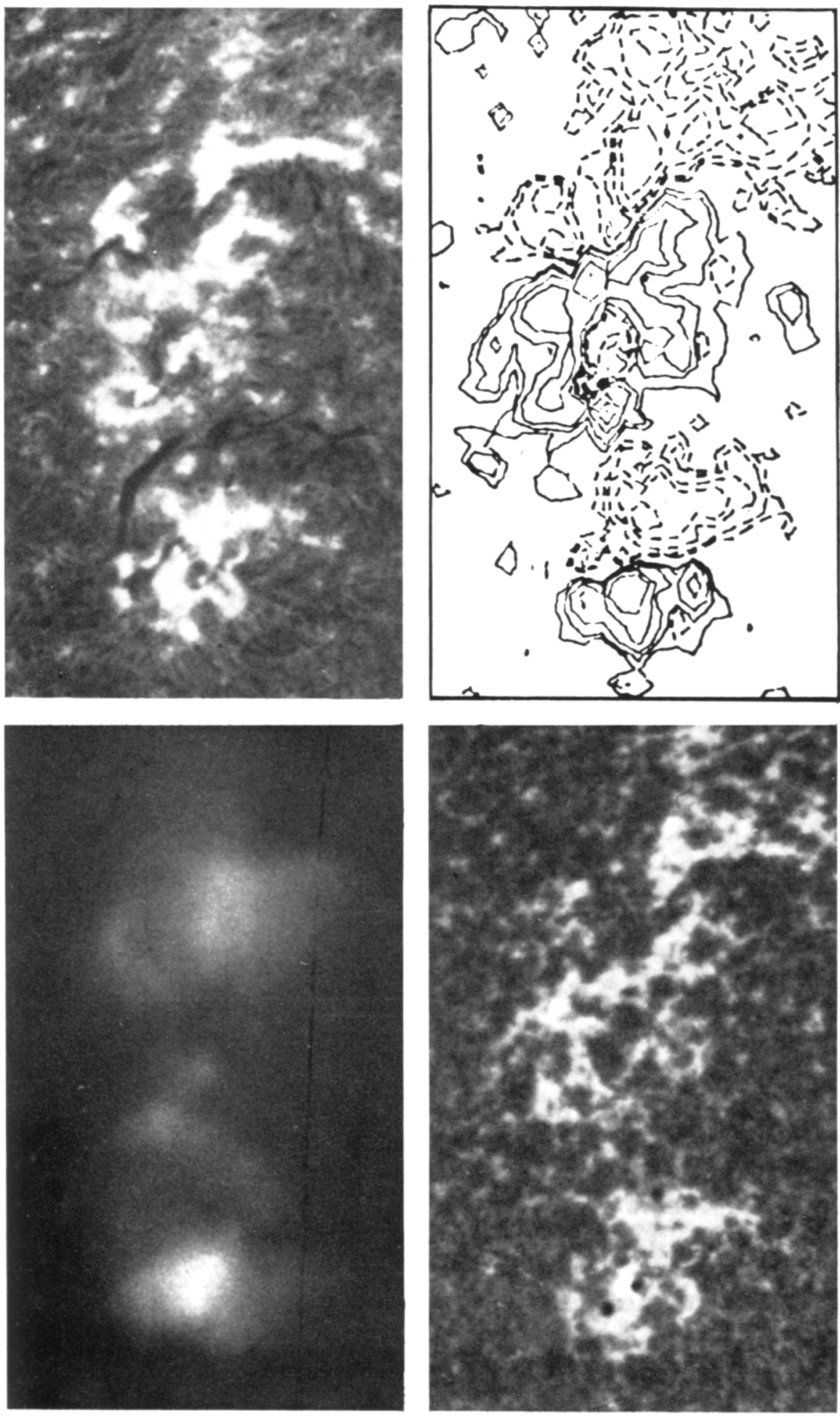

○े

总苍

贯字

艺芒

要它

。ें

Z

马

它

융융

苛.0

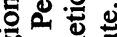

So

踪范

记

政

旸

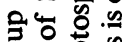

해 중응

$\varangle$

$\because$ 实

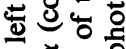
융누 目 궁 웡 อ苋 ธ ธํํㄹ롤 응ํㅀ ชู넌 은 브음

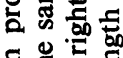
$\Xi$ 告 \&

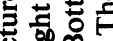
此 㤩领令

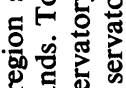
嗼 ०० 尊 视 8 㟧용 踏 สํํㄹ 인 网 告安 m.< o) 记 
bright core, whose width is unresolvable in our telescope, connects the regions of preceding and following polarity across the neutral line. The ends of the bright X-ray core overlie the areas of maximum $\mathrm{H} \alpha$ and $\mathrm{CaK}$ intensity. The core structure passes directly over the region of reduced intensity in $\mathrm{H} \alpha$ and $\mathrm{CaK}$. The X-ray spectrum of the core is harder than that of the rest of the active region when observed through two different passband filters. For example, in the case shown in Figure 3, we have measured the ratio of surface brightnesses of the core, at our resolution, through
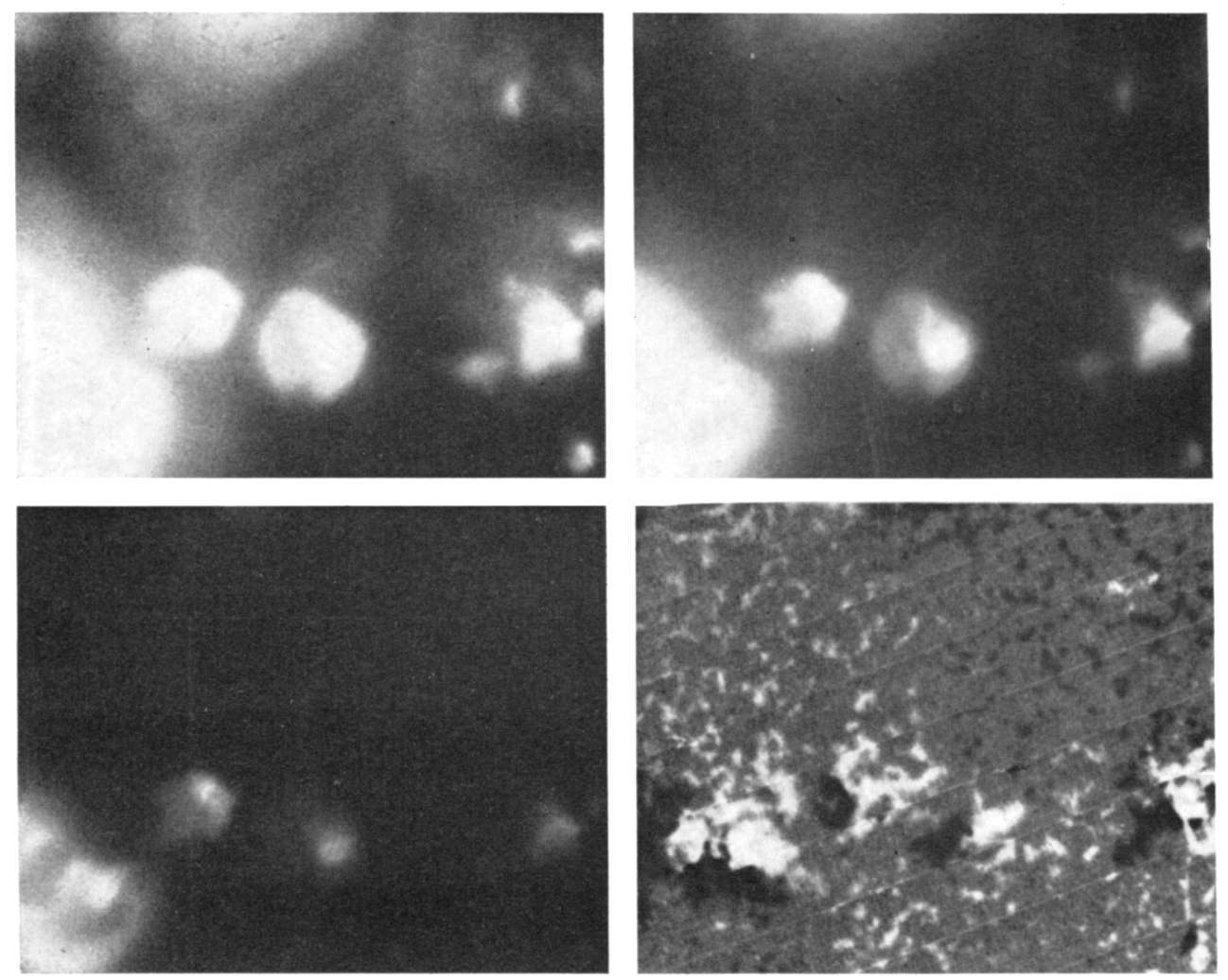

Fig. 4. Comparison of the $X$-ray emitting structure of active regions as observed in three passbands. Top left: $3-36 \AA, 44-64 \AA$. Top right: $3-16 \AA, 44-47 \AA$. Bottom left: $3-13 \AA, 18-24 \AA$. Bottom right: The corresponding photospheric magnetic fields measured by Livingston et al. (1970). The bracket below the photographs is one arc-minute in length.

3-18 $\AA$ and 3-23 $\AA, 44-64 \AA$ waveband filters. It is at least twice that of the remainder of the active region. If one assumes that the $\mathrm{X}$-ray spectrum is of thermal origin, then this implies a higher temperature for the core.

Another group of active regions (Figure 4) is shown in three X-ray passbands (to bring out different aspects of the coronal structures) along with the longitudinal magnetic field. In the softest wavelengths we observe interconnections between the 
active regions as well as the interconnections between portions of the same active region observed in the shorter wavelength exposures. At the shortest wavelengths we note several of the bright core structures. The configurations of the X-ray structures indicate the complexity of the magnetic field pattern. We point out the presence of high field gradients induced by the incursion of following polarity into areas of preceding polarity or vice versa at the sites of some of the bright X-ray cores. Also note that the tubes of coronal plasma interconnecting preceding and following polarities are bent out of the vertical plane. This effect is especially noticeable in the lower left corner of Figure 4, (see also Figure 1). One result of the bending of the X-ray structures is a projection effect which, in the upper left photograph of Figure 4, is noticed as a non-uniform brightness across the active regions. Examination of the shorter wavelength exposures (upper right, and lower left) clarifies the situation.

\section{Groups of Active Regions}

High sensitivity, long wavelength X-ray exposures reveal interconnections between active regions covering extensive areas of the solar disk. One such set of interconnections, on the eastern portion of the disk in Figure 1, covers about $60^{\circ}$ of solar longitude and about $90^{\circ}$ of latitude. The structures between the active regions are 5 to $10 \%$ as bright as the brightest of the active regions they interconnect.

Examination of the underlying photospheric fields indicates that, just as within individual active regions, the interconnections link opposite longitudinal magnetic polarities. Moreover, connections between active regions on opposite sides of the solar equator, link the preceding polarities of the two hemispheres, not the following

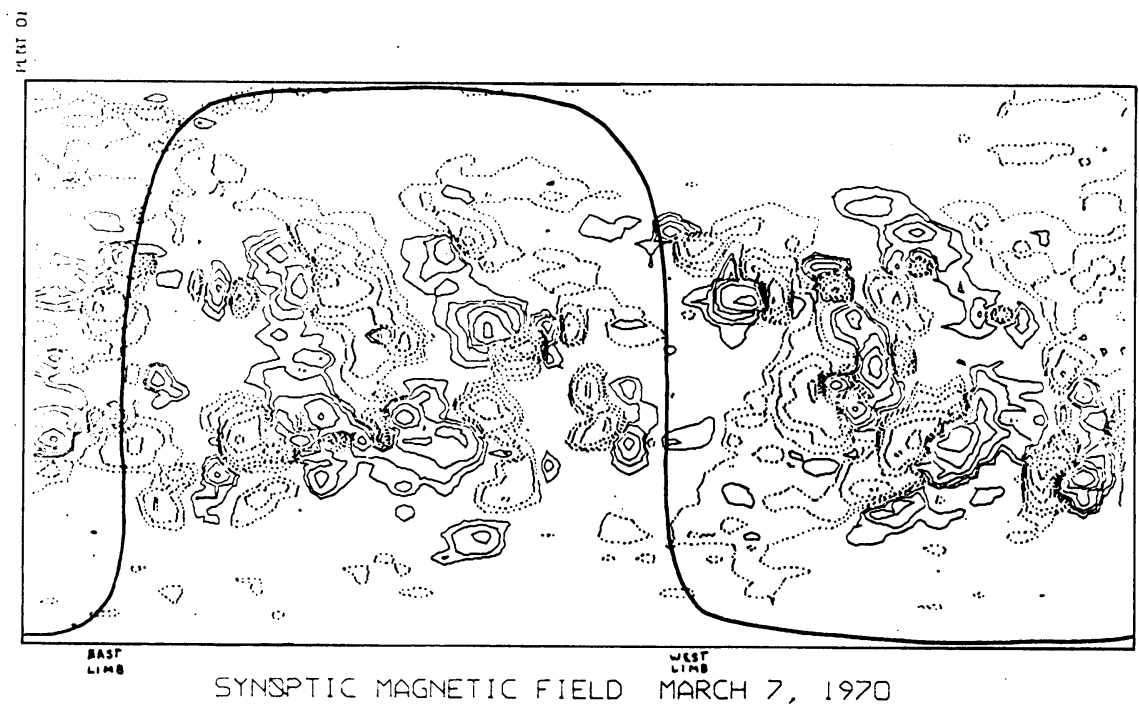

Fig. 5. A synoptic chart of the photospheric magnetic field on March 7, 1970 (from Livingston et al., 1970). 
polarities. Figure 4, a detail of Figure 1, shows bridges crossing the equator which commence over the preceding portions of three southern hemisphere active regions. We interpret these interconnections as indications of the severing and reconnection of the magnetic fields of the active regions as postulated by Babcock (1961).

The active regions are joined into 'complexes of activity' as observed in the magnetograms of Bumba and Howard (1965). Figure 5 is a synoptic chart of the solar magnetic field on March 7, 1970, the date of the rocket flight, as observed by Livingston et al. (1970). It shows that the coronal interconnections which we observe delineate the areas of related photospheric fields. In the areas underlying coronal regions where no interconnections are observed, the average photospheric field appears to be reduced. In Figure 1, two complexes of $\mathrm{X}$-ray interconnection are sharply delimited by a region from which no X-ray emission was observed somewhat to the west of the central meridian. A large dark filament underlies this boundary in the chromospheric spectroheliograms, and the average longitudinal photospheric magnetic fields are significantly weaker there.

The eastern of the two complexes observed on the disk may be identified with a zone of activity. The active regions comprising this complex can be traced back in the CaK reports published in Solar Geophysical Data (1970) for at least 10 rotations. The members of the western group of regions, which also show in terconnections across the solar equator, are only two or three rotations old. These new active regions have formed in the magnetic remnants of older regions. The coronal interconnections that we observe in X-rays may be a manifestation of older features than the active regions presently forming the complex.

\section{X-Ray Emission from the Quiescent Corona}

Faint patches of diffuse X-ray emission are observed on quiescent portions of the solar disk in long exposures with filters having long wavelength X-ray passbands. An example of these features can be seen in Figure 1. In the 44-50 $\AA$ waveband these patches are approximately $\frac{1}{2}$ as bright as the peak limb brightening. Within the errors in our determination, these patches have the same general X-ray spectral distribution as the limb brightening. We conjecture that in fact the patches of soft X-ray emission seen on the disk are regions of enhanced density in the quiet corona.

Figure 6 shows such an area of X-ray emission along with the underlying chromospheric structure seen in $\mathrm{CaK}, \mathrm{H} \alpha$, and the longitudinal component of the solar magnetic field. It is evident that the X-ray emission is associated with an area of increased photospheric magnetic field in the general corona. The X-ray emission of Figure 6 overlies a unipolar magnetic region and its 'ghost' (Bumba and Howard, 1965). The patches of X-ray emission are associated with enhanced areas of the CaK network but they appear to be more diffuse than the corresponding chromospheric features. This is the phenomenon that one would expect if the magnetic field in the corona were to spread from the supergranule boundaries to fill in the supergranule. At the line where a filament is observed in $\mathrm{H} \alpha$ and $\mathrm{CaK}$ separating the unipolar 

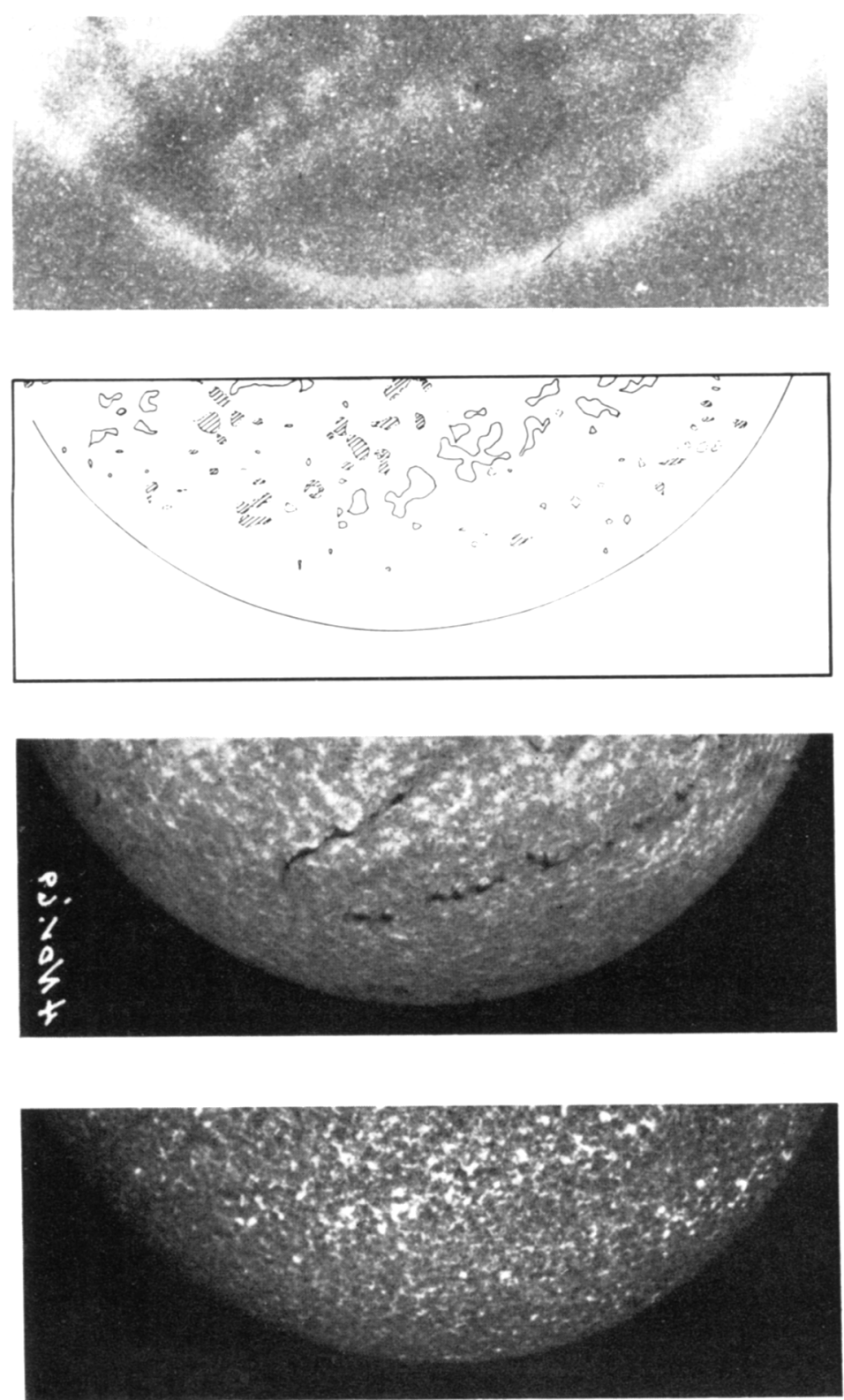

Fig. 6. The appearance of a unipolar magnetic region in (from top to bottom): 3-23 $\AA, 44-64 \AA$ $\mathrm{X}$-rays; photospheric magnetic field (courtesy of Mt. Wilson Observatory); $\mathrm{H} \alpha$ and $\mathrm{CaK}$. The $\mathrm{H} \alpha$ and $\mathrm{CaK}$ photographs were provided by the Sacramento Peak Observatory. 
magnetic region from its ghost, no X-ray emission is apparent. We take these facts to indicate that over the unipolar region and ghost region the coronal density is enhanced, whereas it is reduced over the filament region. Perhaps, it is the coronal material which should have filled this region which has formed the filament. In any case, the UMR delineates a region of enhanced density in the lower corona. When areas which appear on the disk as diffuse coronal emission reach the limb, they are observed as enhancements of the limb brightening. An X-ray photograph obtained in conjunction with the March 7, 1970 solar eclipse was compared with a radial density

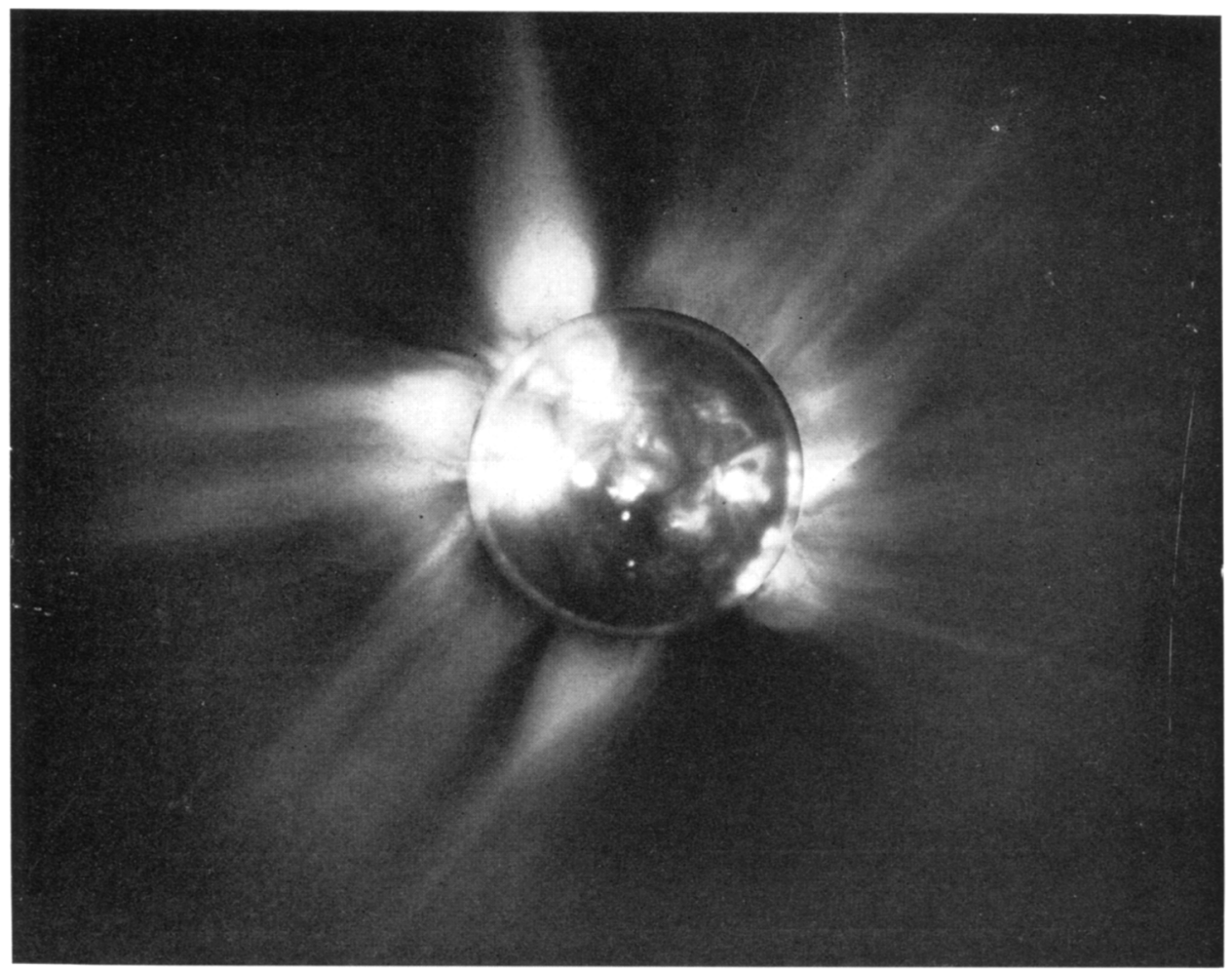

Fig. 7. A composite print of the solar corona on March 7, 1970 composed of an X-ray exposure in the 3-36 $\AA, 44-64 \AA$ wavebands and the radial density gradient filter white light exposure of Newkirk and Lacey (1970).

gradient photograph of the white light corona (Newkirk and Lacey, 1970). There was a one to one correspondence between enhanced X-ray limb brightening and the bases of white light coronal rays and helmet streamers (Van Speybroeck et al., 1970). This fact is evident in a composite print of the X-ray and white light exposures (Figure 7).

Interspersed in the diffuse patches of X-ray emission on the disk are relatively intense spots of brighter emission. These are most noticeable in Figure 8 . They appear to be somewhat higher in temperature than the rest of the general corona on 
the basis of comparisons of exposures with different filters. These point-like regions are associated with all small bipolar areas in the general magnetic field (Figure 1 and Figure 9) and the Ca network is enhanced at their sites. Both from their relative X-ray brightness, and from their bipolar nature, it seems reasonable to assume that these points represent low lying closed structures in the general coronal field. It is possible

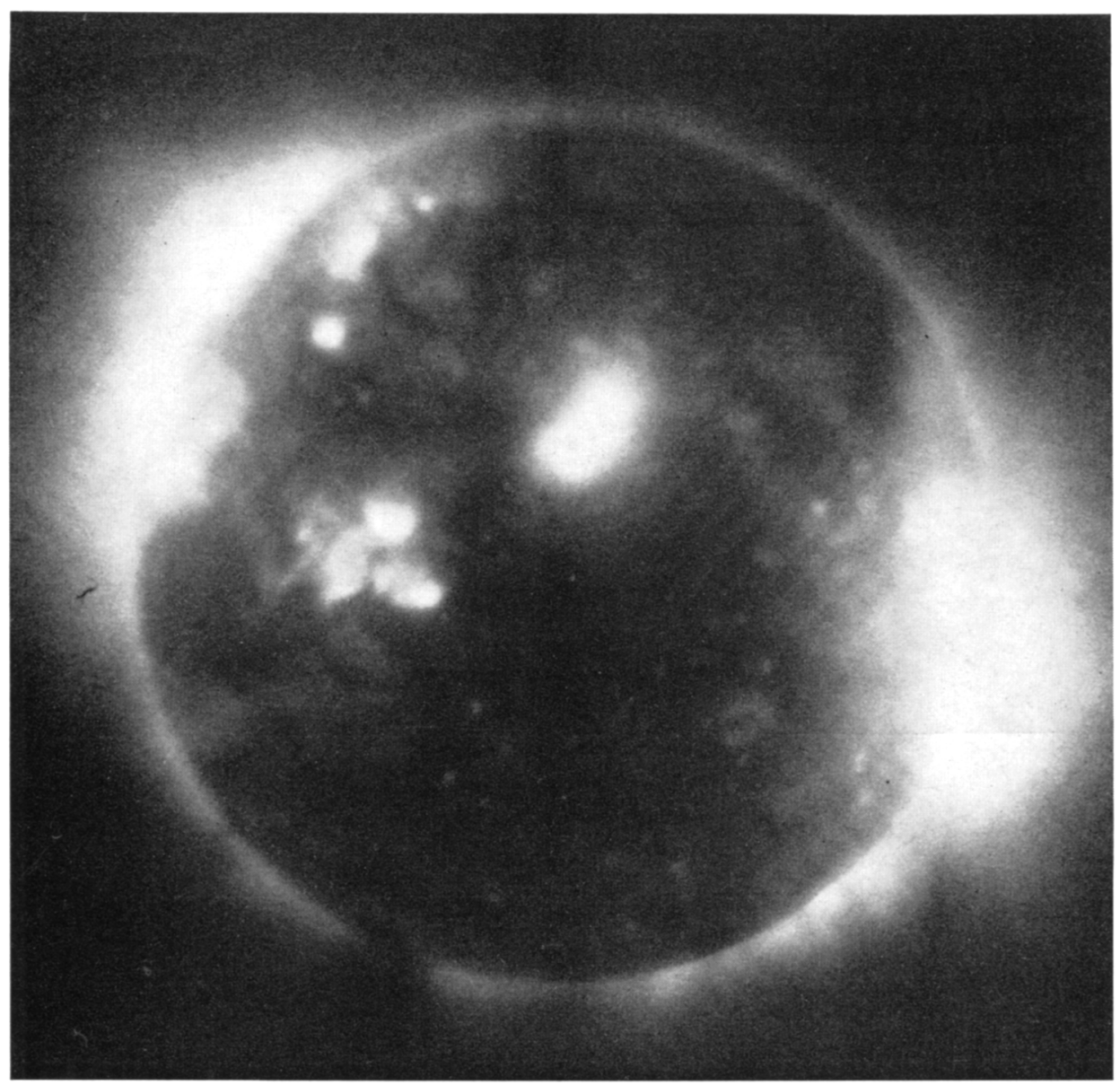

Fig. 8. A photograph of the Sun in the 3-18 $\AA, 44-46 \AA$ wavebands obtained on April 8, 1969.

that they are regions of particularly intense coronal heating or that they are miniature active regions. Further investigation of these features is required.

\section{Flares}

On two of our four rocket flights (June 8, 1968 and November 4, 1969) we have observed importance 1 solar flares in progress. Figure 10 shows a comparison of the 
appearance of the flare observed June 8,1968 in 3 to $18 \AA$ X-rays, $\mathrm{H} \alpha$, and CaK along with a magnetogram of the region in which the flare took place. As we have noted elsewhere (Vaiana and Giacconi, 1969), the X-ray emission follows the form of the $\mathrm{H} \alpha$ emission in general with the important exception of a bright spot bridging the magnetic neutral line of the flare. The bright spot emits more than $50 \%$ of the total energy observed from the flare in the 3-18 $\AA$ passband.

The X-ray appearance of the flare observed on November 4, 1969, seems very different from its appearance in on-band $\mathrm{H} \alpha$, however, as is shown in Figure 11. The $\mathrm{X}$-ray emission, or at least $98 \%$ of the total energy in the 3-18 $\AA$ band, is produced in two regions narrower than the resolution of the telescope $(\sim 4 \mathrm{arcsec})$ and no more than 10 or 15 arc sec long. The two spots of X-ray emission correspond to the region of brightest emission in the wings of $\mathrm{H} \alpha$. We therefore interpret these X-ray spots as
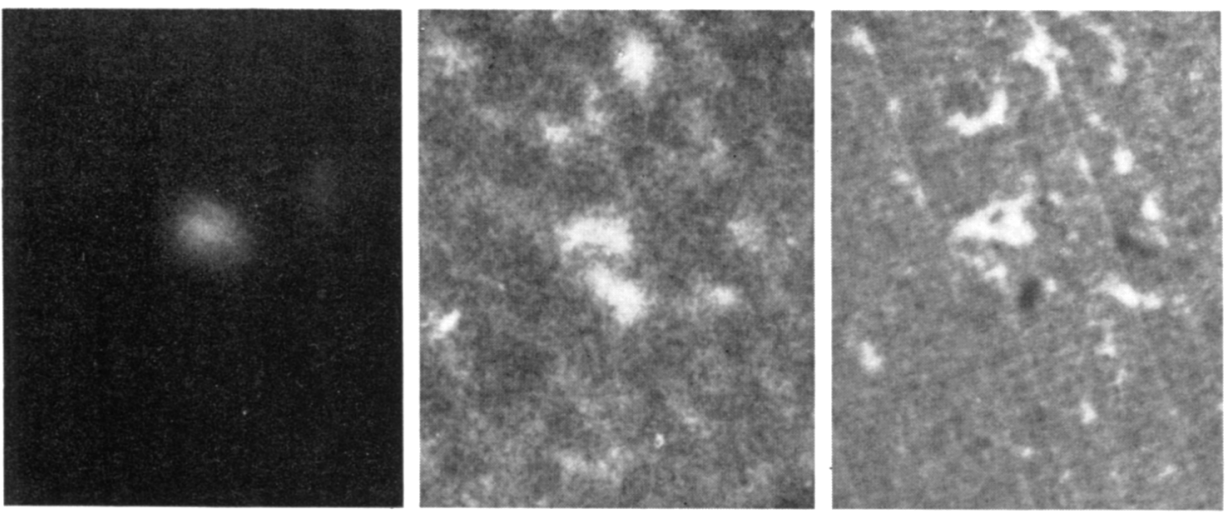

Fig. 9. An X-ray 'bright point' in the quiescent corona and the underlying structures in CaK. (courtesy of Sacramento Peak Observatory) and the photospheric magnetic field (Livingston et al., 1970). The bracket below the photographs is one arc minute in length.

being asociated with the hottest points in the $\mathrm{H} \alpha$ flare. Unfortunately, magnetic field information was not available for this region for times close to the flare, because the flare was near the limb. However, we note that in the 3-12 \& waveband the June 8, 1968 flare appears strikingly similar to the November 4, 1969 flare (Figure 12) in that the majority of the emission is from a restricted region. It would appear that the two bright spots observed on November 4, 1969 are analogous to the bright core of the June 8, 1968 flare although flare X-ray emission from the rest of the X-ray plage at longer wavelengths was substantially weaker.

The bright cores which we have recently observed in non-flaring active regions bear a strong resemblance to the situation observed during the June 8,1968 flare despite the difference of a factor of 50 to 100 in the surface brightness of flares and active regions. Both the cores of active regions and the spot of most intense emission in the flare are narrow linear structures bridging the neutral line of the longitudinal field at a point of 
high gradient. With regard to the November 4, 1969 flare the situation is less clear because of the lack of magnetic field data and the position of the flare near the limb. It might be that the two spots of flare X-ray emission bridge the neutral line at two nearby points as we have occasionally observed in active regions (Figure 4). Alternatively, it might be that these spots which are associated with the region of maximum $\mathrm{H} \alpha$ emission are analogous to the foot points of the active region core. It may be
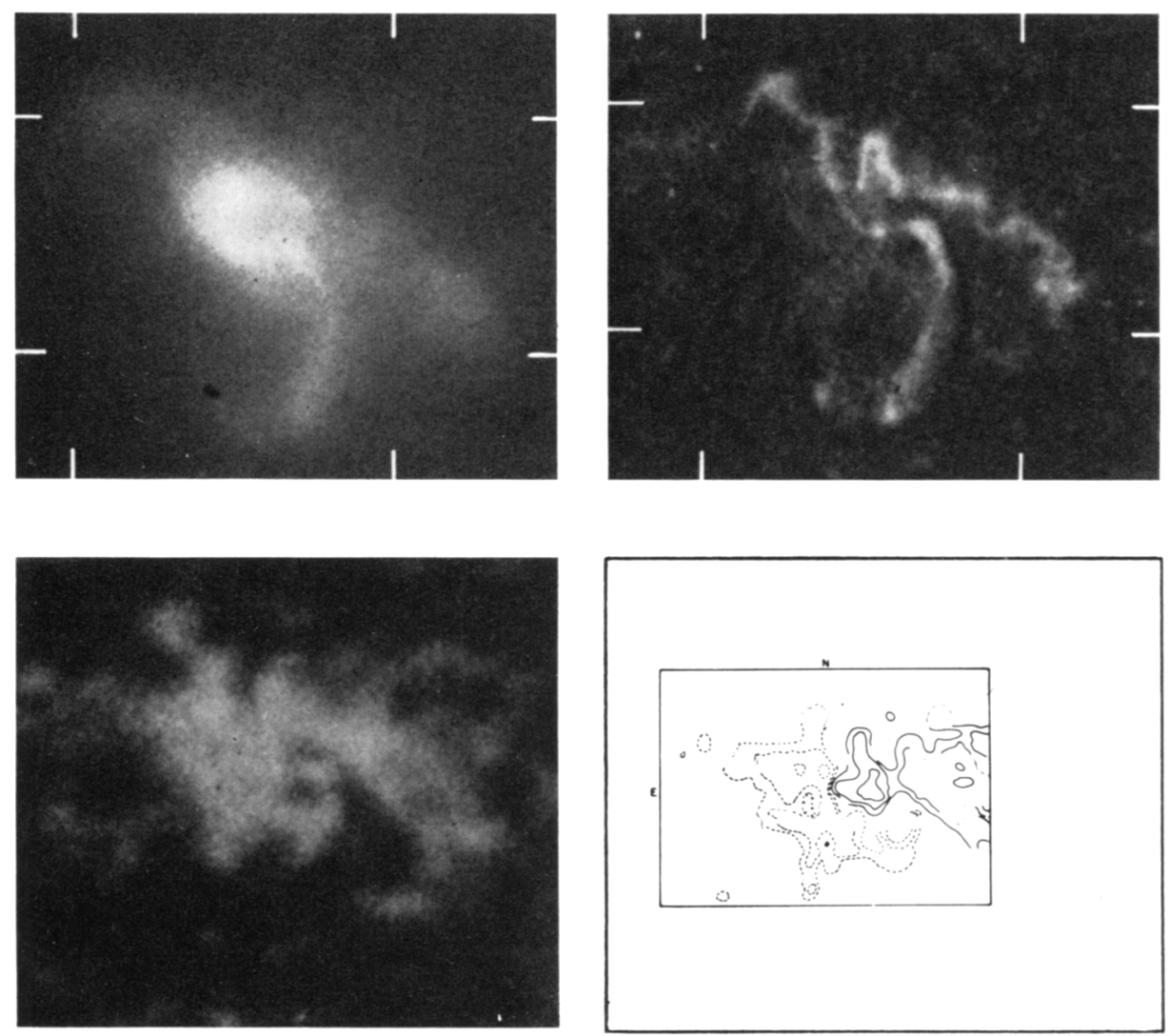

Fig. 10. Appearance of the solar flare of June 8, 1968 (1742 UT) in X-rays, $\mathrm{H} \alpha$ and $\mathrm{CaK}$ and the magnetic field configuration at the time of the flare. Top left: $3-18 \AA \mathrm{X}$-rays. Top right: $\mathrm{H} \alpha$ (courtesy of ESSA Boulder Observatory). Bottom left: CaK (courtesy of McMath-Hulbert Observatory). Bottom right: Photospheric longitudinal field (courtesy of J. Harvey, Kitt Peak National Observatory). The bracket beside the photographs is one arc-minute in length.

highly significant that the June 8 observations took place at the peak of the flare while a non-thermal radio burst was in progress. On the other hand, the November 4 observations were made during the decay phase of the flare. In any case, our observations suggest that the bright, X-ray core of the active region becomes the site of the most intense X-ray emission in flares. The bright X-ray core may play an important role in the flare process. 

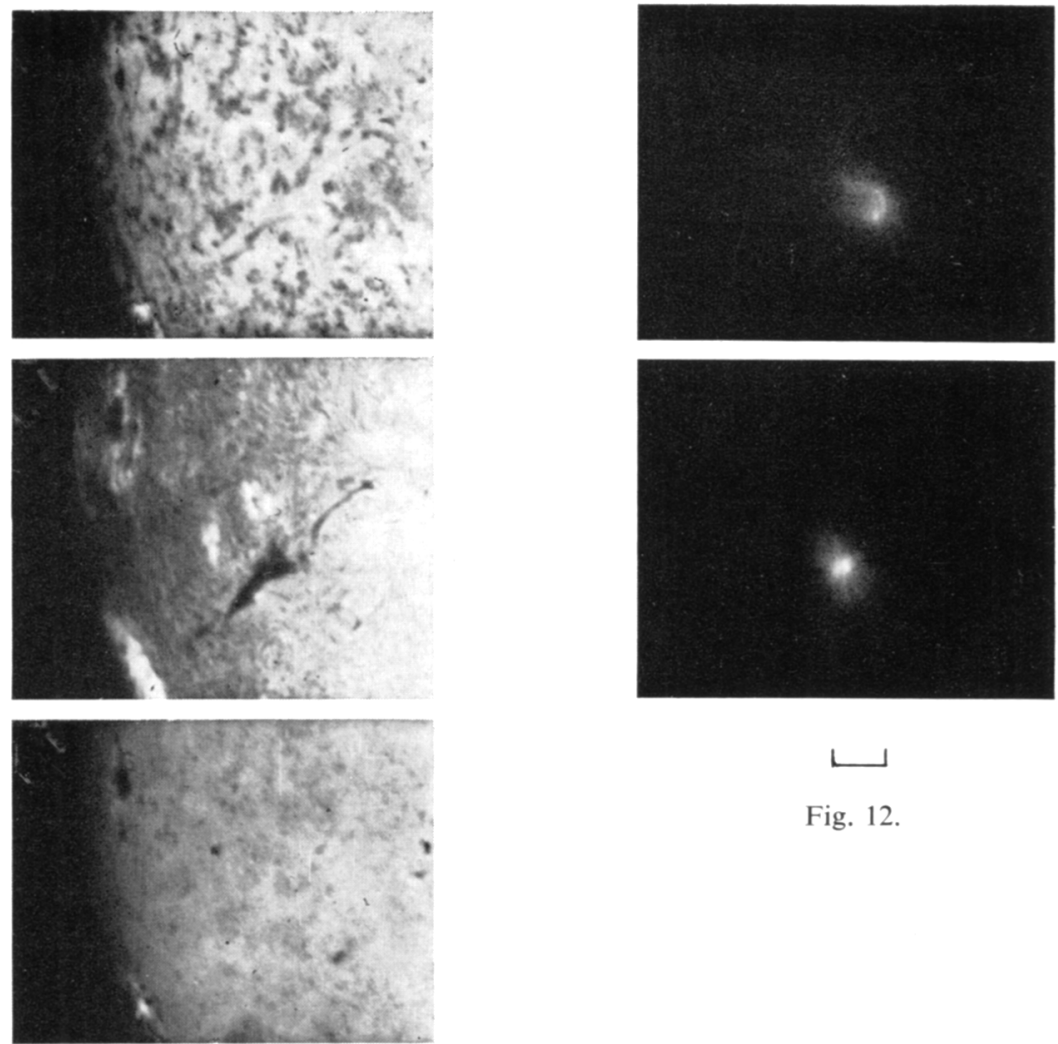

Fig. 12.

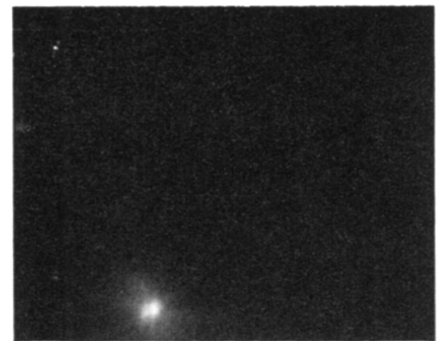

Fig. 11 .

Fig. 11. The appearance of the solar flare of November 4, 1969 in H $\alpha$ and X-rays. Top to bottom: Red wing $\mathrm{H} \alpha$; On-band $\mathrm{H} \alpha$; Blue wing $\mathrm{H} \alpha$; 3-12 $\AA \mathrm{X}$-rays. The photographs were taken at about 2030 UT. The $\mathbf{H} \alpha$ photographs were provided by H. Zirin, California Institute of Technology. The bracket below the photographs is one arc minute in length.

Fig. 12. A comparison of the appearance of the June 8, 1968 flare (top) and the November 4, 1969 flare (bottom) in the 3-12 $\AA$ waveband. The bracket below the photographs is one arc minute in length. 


\section{Conclusions}

Direct comparisons of the soft X-ray images of the corona with the photospheric magnetic fields indicate that the X-ray structures do indeed trace the configuration of the magnetic field through the lower corona. It appears that given sufficient sensitivity and resolution X-ray emitting coronal structures can be observed whereever there is magnetic field penetrating the corona. A range of phenomena have been observed showing field lines which close at a variety of heights in the solar atmosphere.

Active regions appear to be composed of tubular structures often taking the form of loops or arches, which have their feet in regions of opposite magnetic polarity. These loops usually interconnect portions of the same or adjacent active regions.

Higher, larger structures link widely separated active regions into complexes of activity covering substantial fractions of the disk. The complexes are separated by areas of low average longitudinal field in the photosphere and by quiescent filaments in the chromosphere. Interconnections across the solar equator appear to originate over areas of preceding polarity.

Enhanced X-ray emission is observed, outside the active belt, over unipolar magnetic regions. The X-ray features appear more diffuse than the underlying chromospheric network. Reconnection of these field lines takes place high in the corona where the structures can only be observed in white light. Bright point-like X-ray features are observed above bipolar areas in the general coronal field. Presumably these indicate structures which reconnect low in the corona.

Two, probably related, classes of X-ray features are associated with points of high field gradient along the longitudinal magnetic, 'neutral line'. Both in the non-flaring active region and in flares the brightest emission is observed from one or more line sources (at our present resolution) associated with the 'neutral line' at the point of maximum gradient. In flares this can account for a substantial fraction of the total soft X-ray emission of the flare.

To date, only four rocket flights of the high resolution grazing incidence X-ray telescope have been conducted. The total observation time involved is about $15 \mathrm{~min}$. Despite the limited number of observations, X-ray imaging has obtained new information on the configuration of the lower corona under conditions ranging from flares to unipolar magnetic regions. Each of the rocket flights has revealed at least one phenomenon that had not been observed before and that had not been anticipated.

\section{Acknowledgements}

The group of investigators who have participated in the solar X-ray rocket program includes, in addition to the authors, R. Giacconi, W. Reidy, T. Zehnpfennig, J. Davis and M. Zombeck. This work was supported by NASA under contracts NASW-2027 and NAS5-9041. 


\section{References}

Babcock, H. W.: 1961, Astrophys. J. 133, 572.

Bumba, V. and Howard, R.: 1965, Astrophys. J. 141, 1502.

Giacconi, R., Reidy, W., Vaiana, G., VanSpeybroeck, L., and Zehnpfennig, T.: 1969, Space Sci. Rev. 9,3.

Livingston, W., Harvey, J., and Slaughter, C.: 1970, Nature 226, 1146.

Newkirk, G. and Lacey, L.: 1970, Nature 226, 1098.

Solar-Geophysical Data Bulletin: 1970, Environmental Science Services Administration, U.S. Dept. of Commerce, Boulder, Colo., IER-FB-309.

Vaiana, G., Reidy, W., Zehnpfennig, T., VanSpeybroeck, L., and Giacconi, R.: 1968, Science 161, 564.

Vaiana, G. and Giacconi, R.: 1969, in Plasma Instabilities in Astrophysics, (ed. by D. A. Tidman and D. G. Wentzel), Gordon and Breach, N.Y., p. 91.

Van Speybroeck, L. P., Krieger, A. S., and Vaiana, G. S.: 1970, Nature 227, 818. 